\title{
Regulation of the Reacted Layer Thickness in a Gas-Solid Reacting System
}

\author{
Miguel A. Barron1, Dulce Y. Medina1, Gabriel Plascencia² \\ ${ }^{1}$ Departamento de Materiales, Universidad Autonoma Metropolitana Azcapotzalco, Mexico, D.F., Mexico \\ ${ }^{2}$ Centro de Investigacion e Innovacion Tecnologica-IPN, Mexico, D.F., Mexico \\ Email: bmma@correo.azc.uam.mx
}

Received 18 April 2014; revised 25 May 2014; accepted 10 June 2014

Copyright (C) 2014 by authors and Scientific Research Publishing Inc.

This work is licensed under the Creative Commons Attribution International License (CC BY).

http://creativecommons.org/licenses/by/4.0/

(c) (7) Open Access

\begin{abstract}
A mathematical model for the analysis of a gas-solid reacting system is presented. This model is an alternative to the classical shrinking-core model. The model has a structure that can be easily transformed into a canonical control form, which is proper for controller synthesis. Analytical solution of the model to describe the open-loop behavior is expressed in terms of the Lambert function. The Lambert function is evaluated from a Taylor expansion series. Besides, a controller is proposed to regulate the reacted layer thickness using initially the diffusion coefficient as control input. The control law is synthesized employing the feedback linearization technique. Main contributions of this work are the synthesis of the layer thickness controller, and the employment of the process temperature as substitute of the diffusion coefficient as the control input.
\end{abstract}

\section{Keywords}

Diffusion Coefficient, Feedback Linearization, Gas-Solid System, Lambert Function, Reacted Layer, Thickness Regulation

\section{Introduction}

In the extractive metallurgy industry, gas-solid reactions are very common, for example the reduction of iron ore with carbon monoxide, the roasting of copper ores, or the combustion of coke. The shrinking core model (SCM) [1] has been employed long time ago to analyze the occurring reactions and to design the equipment where these reactions take place. Unfortunately, mathematical solution of the SCM is very complex, particularly for the case in which the thickness of the involved layers is moving and variable [2]. A less complex alternative to the SCM is found in [3] [4], where a relatively simple mathematical model is proposed for the analysis of gas-solid reacting systems with moving boundaries. The mathematical model reported in [4] is described in this work, and an 
open-loop solution obtained using the Taylor expansion series of the Lambert function is presented and discussed.

On the other hand, from operative and economical points of view it is imperative to have a tight control of the gas-solid reacting systems in order to guarantee the product quality, reduce the residence time and costs, and optimize the design and operation of the industrial equipment. Therefore, an automatic control strategy to regulate the thickness of the reacted layer in a gas-solid reacting system is required. In this work a controller is synthesized using the mathematical model reported in [4]. The feedback linearization technique is employed for the controller synthesis and the diffusion coefficient is initially chosen as the control input. Temperatures corresponding to the diffusion coefficients are obtained from an empirical expression, allowing the usage of a more easily manipulable variable in the control algorithm. Dynamic performance of the closed-loop system is illustrated by means of numerical simulations. This work is organized as follows: in Section 2 the mathematical model is presented; in Section 3 the open-loop results are described; in Section 4 the controller synthesis is explained; in Section 5 the closed-loop results are discussed; and finally, the conclusions are presented in Section 6.

\section{Mathematical Model}

Consider a flat solid surface immersed in a gaseous environment. The gas chemically reacts with the solid and enters into the solid by diffusion through the reacted layer. In [3] [4] is assumed that the total thickness $X$ of the surface is moving and variable and depends linearly on time $t$ as follows

$$
X(t)=L_{0}-R t
$$

where $L_{0}$ is the initial thickness and $R$ is a parameter which defines the chemical reaction rate. $X$ is increased or decreased depending on the value of $R$ : if $R<0$ swelling occurs, and if $R>0$ shrinking occurs. Besides, a mass balance yields

$$
Z(t)=X(t)-Y(t)
$$

where $Y$ is the thickness of the unreacted layer and $Z$ is the thickness of the reacted layer.

The rate of change of $Y$ is directly proportional to the rate of diffusion of the gas through the reacted layer, and inversely proportional to $Z$ :

$$
\frac{\mathrm{d} Y}{\mathrm{~d} t}=-\frac{D(T)}{Z(t)}
$$

In the above equation $D$ is the diffusion coefficient of the gas through the solid, and $T$ is the process temperature. Equation (3) and the time derivatives of Equations (1) and (2) yield the rate of change of the reacted layer:

$$
\frac{\mathrm{d} Z}{\mathrm{~d} t}=-R+\frac{D(T)}{Z(t)}
$$

At steady state $\mathrm{d} Z / \mathrm{d} t=0$, and from Equation (4) the equilibrium value of the reacted layer thickness $Z_{e}$ is given by

$$
Z_{e}(T)=\frac{D(T)}{R}
$$

\section{Model Solution and Open-Loop Results}

Using the variables separation method, the analytical solution of Equation (4) is [4]

$$
Z(t)=\frac{D}{R}\left[1+W\left(-\exp \left(-1-\frac{R^{2}}{D} t\right)\right)\right]
$$

where $W$ is the Lambert function. $W$ has curious mathematical properties and interesting applications; it is highly nonlinear, and some of its properties are as follows: $W(0)=0, W\left(-\mathrm{e}^{-1}\right)=-1$ [5]. An additional property of $W$ is [6] 


$$
W(x) \mathrm{e}^{W(x)}=x
$$

The open-loop time evolution of $Z$, i.e. the reacted layer thickness, is obtained through Equation (6). The required Lambert function is evaluated from Equation (7), which is solved using the first ten terms of the Taylor series expansion [7]

$$
W(x)=\sum_{n=1}^{\infty} \frac{(-1)^{n-1} n^{n-2}}{(n-1) !} x^{n}
$$

or applying the iterative first order Newton-Raphson method [8]. Both procedures yielded identical results.

Once obtained $Z(t)$ from Equation (6), $X(t)$ is determined from Equation (1), and $Y(t)$ is calculated from Equation (2). Results are shown in Figure 1 for the following values of parameters reported in [4]: $L_{0}=300 \mu \mathrm{m}, R=$ $0.001 \mu \mathrm{m} / \mathrm{s}, D=0.1 \mu \mathrm{m}^{2} / \mathrm{s}$.

Using the above parameter values in Equation (5) yields $Z_{e}=100 \mu \mathrm{m}$. Figure 1 shows that the reacted layer progressively increases from an initial value of 0 to a final value of $Z_{e}$. The unreacted layer decreases from its initial value of $L_{0}$ to a final value of 0 . On the other hand, the total layer thickness decreases from an initial value of $L_{0}$ to a final value $X_{e}=Z_{e}$. These results are consistent with the expected behavior of gas-solid shrinking reacting systems with $R>0$. The equilibrium time $t_{e}$ required for reaching the equilibrium state is obtained from Equation (1) considering that in this condition $X_{e}=Z_{e}$ :

$$
t_{e}=\frac{L_{0}-Z_{e}}{R}
$$

With the above values of $L_{0}, Z_{e}$ and $R$, Equation (9) yields $t_{e}=200,000 \mathrm{~s}$. This time coincides with that required for thickness stabilization of the reacted, unreacted and total layers in the open-loop results of Figure 1 obtained through the Lambert function.

\section{Controller Synthesis}

For the controller synthesis, feedback linearization technique is employed [9] [10]. Given that the control objective is the regulation of the reacted layer thickness, $Z$ becomes the control output. The chosen manipulated variable is the diffusion coefficient, which depends on the temperature; then $D$ is selected as the control input. The output error is defined as

$$
e(t)=Z(t)-Z_{e}
$$

where $Z_{e}$ is selected as the control set point. An asymptotically stable behavior is imposed to the error:

$$
\dot{e}+k e=0
$$

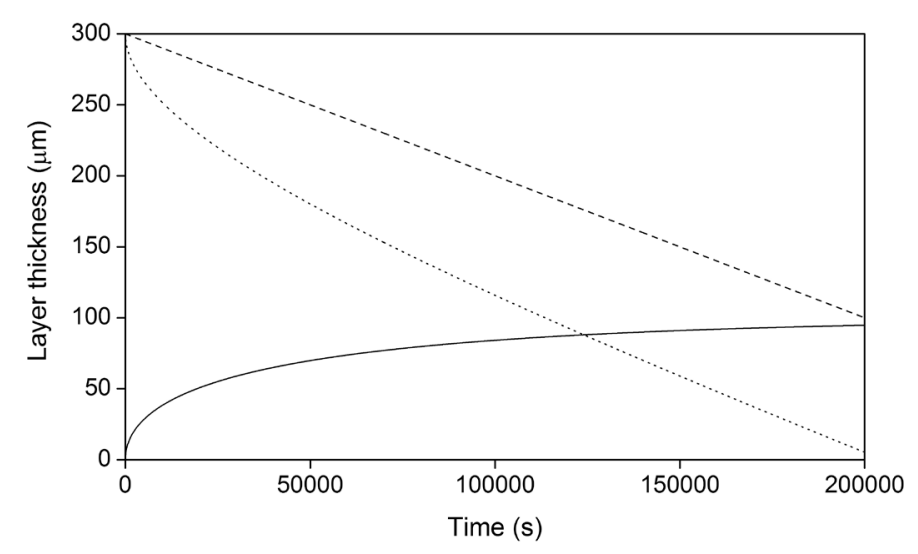

Figure 1. Open-loop time evolution of the layers thickness. Reacted (solid), unreacted (dotted), total (dashed). 
where $k$ is the control gain. This behavior assures that

$$
\lim _{t \rightarrow \infty} e(t)=0
$$

Therefore

$$
\lim _{t \rightarrow \infty} Z(t)=Z_{e}
$$

Considering the output error definition, and substituting it into Equation (4), the following control law arises

$$
D(Z)=Z\left[R-k\left(Z-Z_{e}\right)\right]
$$

From Equation (13) the steady state value of the control input is given by $D_{e}=Z_{e} R=0.1 \mu \mathrm{m}^{2} / \mathrm{s}$. The characteristic time $\tau$ of the gas-solid system is given by [4]

$$
\tau=\frac{D_{e}}{R^{2}}
$$

Then, $\tau=1 \times 10^{5} \mathrm{~s}$. A proper value of the control gain is $k=1 / \tau=1 \times 10^{-5} \mathrm{~s}^{-1}$. To speed up the closed-loop dynamics, in the numerical simulations described later a value of $k=5 \times 10^{-5} \mathrm{~s}^{-1}$ is selected.

\section{Closed-Loop Results}

Closed-loop dynamics is obtained by numerical integration of Equation (4) employing the fourth order RungeKutta method [8] with time step of $1 \times 10^{-4} \mathrm{~s}$, and considering the control law defined by Equation (13).

Figure 2 shows the dynamics behavior of the reacted layer thickness, i.e. the control output. As expected, the behavior is asymptotic. The control output reaches the set point in around 130,000 s, whereas for the closed-loop case it takes a time of 200,000 s, as is seen in Figure 1.

The dynamics of the control input, i.e. the diffusion coefficient, is observed in Figure 3. The control input presents a peak of $0.18 \mu \mathrm{m}^{2} / \mathrm{s}$ in around $18,000 \mathrm{~s}$, and reaches its equilibrium value in 130,000 s, i.e. the same time at which the control input arrives to its set point.

Direct utilization of the diffusion coefficient as control input is unrealistic. In current industrial practice, temperature is by far a more easily manipulable variable. Unfortunately, in [4] is not reported the dependence of the diffusion coefficient on the temperature. In [11] the mechanisms of carburization and melting of solid iron by gaseous carbon monoxide is studied. There, the following empirical expression which relates temperature and diffusion coefficient is reported:

$$
D(T)=C_{1} \mathrm{e}^{\left(-\frac{E_{a}}{R_{g} T}\right)}
$$

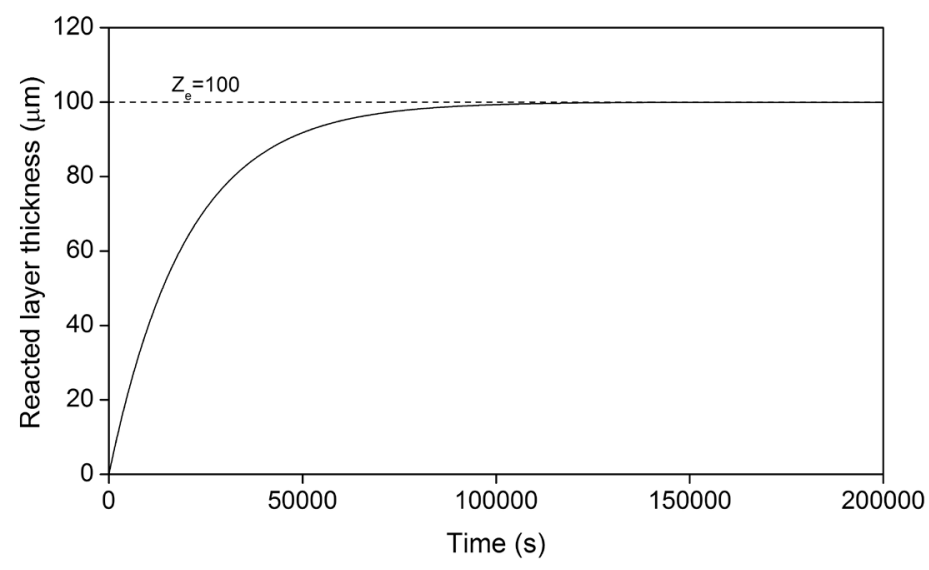

Figure 2. Closed-loop dynamics of the reacted layer thickness. 


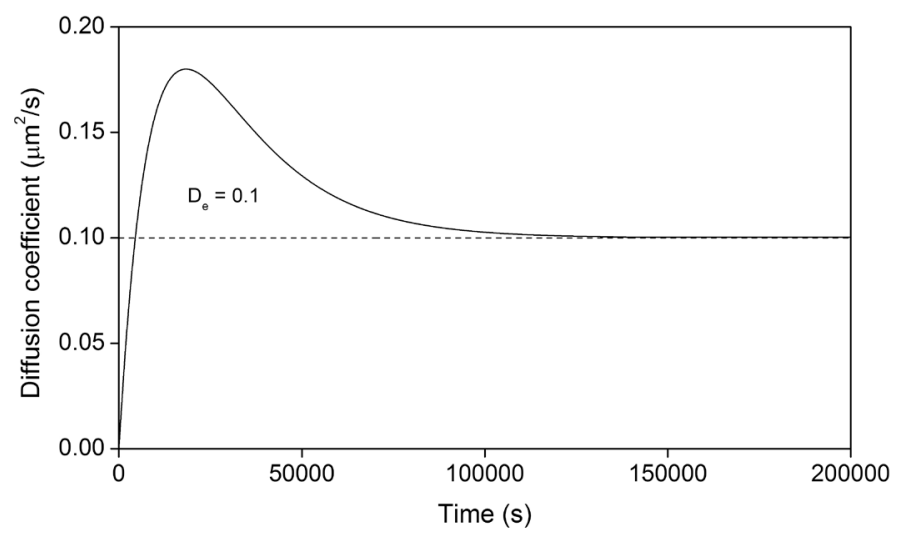

Figure 3. Closed-loop dynamics of the diffusion coefficient.

where $D$ is expressed in $\mathrm{m}^{2} / \mathrm{s}, C_{1}=2 \times 10^{-6}$ is a constant, $E_{a}=1.12 \times 10^{-5}$ is the activation energy of the reaction, and $R_{g}=8.314$ is the universal gas constant. $T(\mathrm{~K})$ is the absolute temperature of the process. Solving the above expression for $\mathrm{T}$ yields:

$$
T=\frac{E_{a}}{R_{g} \ln \left(\frac{C_{1}}{D}\right)}
$$

The new control law in terms of the process temperature is obtained by substitution of Equation (13) into Equation (16):

$$
T(Z)=\frac{E_{a}}{R_{g} \ln \left(\frac{C_{2}}{Z\left[R-\left(Z-Z_{e}\right)\right]}\right)}
$$

where $C_{2}=2 \times 10^{6}$ comes from the unit conversion of the diffusion coefficient from $\mathrm{m}^{2} / \mathrm{s}$ to $\mu \mathrm{m}^{2} / \mathrm{s}$.

Closed-loop steady state temperature $T_{e}$ is obtained from Equation (17) considering that at steady state $Z=Z_{e}$ :

$$
T_{e}=\frac{E_{a}}{R_{g} \ln \left(\frac{C_{2}}{Z_{e} R}\right)}
$$

The process temperatures corresponding to the diffusion coefficients of Figure 3 are determined from Equation (17). Figure 4 shows the obtained temperatures and their closed-loop dynamic behavior. A peak of $830.4 \mathrm{~K}$ at around 18,000 s is reached by temperature. A value of $801.3 \mathrm{~K}$ for $T_{e}$ is obtained from Equation (18) considering that $Z_{e}=100 \mu \mathrm{m}$ and $R=0.001 \mu \mathrm{m} / \mathrm{s}$. This value of $T_{e}$ is corroborated in the results of Figure 4.

\section{Conclusions}

A mathematical model from literature which describes the dynamics of a gas-solid reacting system was presented. This model is a less complex alternative to the classical shrinking core model.

1) Solution to the above model, based on the Taylor series expansion of the Lambert function, was obtained and discussed. Closed-loop time evolution of the layers thickness is in agreement with the expected behavior of gas-solid shrinking reacting systems with moving boundaries.

2) A theoretical time of 200,000 s for achieving the open-loop steady-state was determined for the considered values of parameters. This time is in agreement with that obtained in the open-loop results using the Lambert function.

3) A controller to regulate the thickness of the reacted layer was synthesized using the feedback linearization technique. This controller uses as control input the diffusion coefficient of the gas into the solid. As expected, dynamic closed-loop performance is asymptotically stable under the considered conditions. 


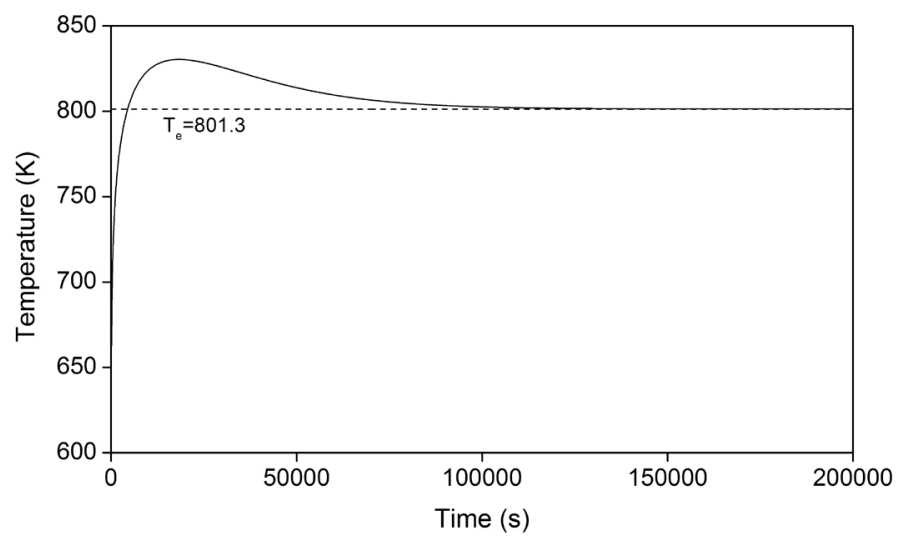

Figure 4. Closed-loop dynamics of the process temperature.

4) Temperature corresponding to the above diffusion coefficients was determined from a previous reported empirical expression. This allows the utilization of a more realistic, easily manipulable variable at industry as temperature in the control algorithm.

\section{References}

[1] Levenspiel, O. (1999) Chemical Reaction Engineering. 3rd Edition, Wiley, Hoboken.

[2] Son, H.Y. and Wadsworth, M.E. (2012) Rate Processes of Extractive Metallurgy. Springer, London.

[3] Braun, A., Bartsch, M., Schnyder, B. and Kotz, R. (2000) A Model for the Film Growth in Samples with Two Moving Reaction Frontiers an Application and Extension of the Unreacted-Core Model. Chemical Engineering Science, 55, 5273-5282. http://dx.doi.org/10.1016/S0009-2509(00)00143-3

[4] Braun, A., Wokaun, A. and Hermanns, H.-G. (2003) Analytical Solution to a Growth Problem with Two Moving Boundaries. Applied Mathematical Modeling, 27, 47-52. http://dx.doi.org/10.1016/S0307-904X(02)00085-9

[5] Jeffrey, D.J., Hare, D.E. and Corless, R.M. (1996) Unwinding the Branches of the Lambert W Function. The Mathematical Scientist, 21, 1-7.

[6] Chapeau-Blondeau, F. (2002) Numerical Evaluation of the Lambert W Function and Application to Generation of Generalized Gaussian Noise with Exponent 1/2. IEEE Transactions on Signal Processing, 50, 2160-2165. http://dx.doi.org/10.1109/TSP.2002.801912

[7] Corless, R.M., Jeffrey, D.J. and Knuth, D.E. (1997) A Sequence of Series for the Lambert W Function. In Proceedings of the 1997 International Symposium on Symbolic and Algebraic Computation, ACM, New York, 197-204. http://dx.doi.org/10.1145/258726.258783

[8] Chapra, S.C. and Canale, R.P. (2010) Numerical Methods for Engineers. 6th Edition, McGraw-Hill, New York.

[9] Slotine, J.J. and Li, W. (1991) Applied Nonlinear Control. Prentice-Hall, Englewood Cliffs.

[10] Khalil, H.K. (2002) Nonlinear Systems. 3rd Edition, Prentice-Hall, Upper Saddle River.

[11] Murakami, T., Fukuyama, H. and Nagata, K. (2001) Mechanisms of Carburization and Melting of Iron by CO Gas. ISIJ International, 41, 416-421. http://dx.doi.org/10.2355/isijinternational.41.416 
Scientific Research Publishing (SCIRP) is one of the largest Open Access journal publishers. It is currently publishing more than 200 open access, online, peer-reviewed journals covering a wide range of academic disciplines. SCIRP serves the worldwide academic communities and contributes to the progress and application of science with its publication.

Other selected journals from SCIRP are listed as below. Submit your manuscript to us via either submit@scirp.org or Online Submission Portal.
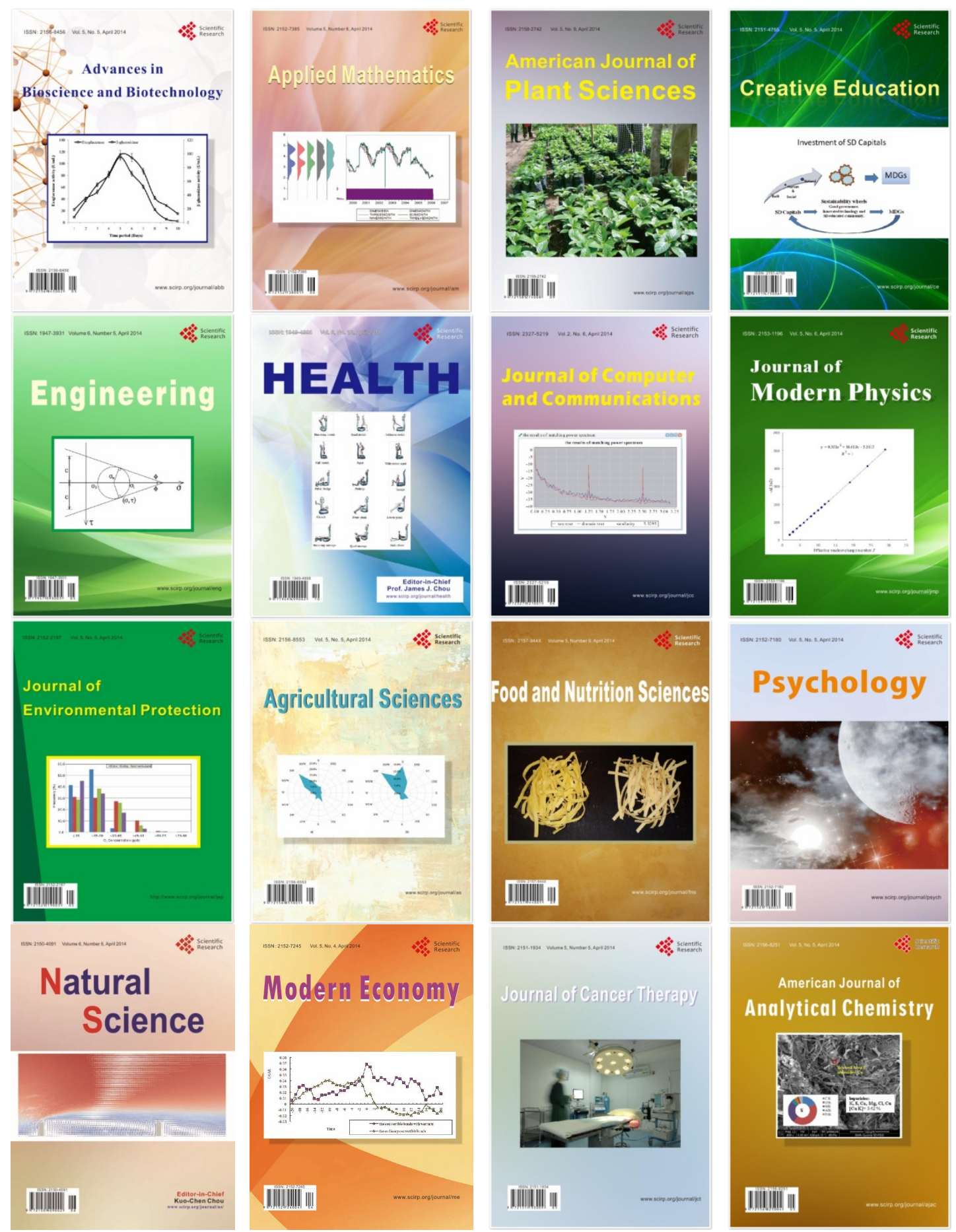